\title{
A 3-year Plan for Beam Science in the Heavy-Ion Fusion Virtual National Laboratory
}

\section{Grant Logan, HIF-VNL Director November 9, 2001}

\section{Introduction}

In December 1998, LBNL Director Charles Shank and LLNL Director Bruce Tarter signed a Memorandum of Agreement to create the Heavy-Ion Fusion Virtual National Laboratory (HIF-VNL) with the purpose of improving the efficiency and productivity of heavy ion research through coordination of the two laboratories' efforts under one technical director. In 1999, PPPL Director Robert Goldston signed the VNL MOA for PPPL's heavy-ion fusion group to join the VNL. LBNL and LLNL each contribute about $45 \%$ of the $\$ 10.6 \mathrm{M} / \mathrm{yr}$ trilab VNL effort, and PPPL contributes currently about $10 \%$ of the VNL effort. The three labs carry out collaborative experiments, theory and simulations of a variety of intense beam scientific issues described below. The tri-lab HIF VNL program is part of the DOE Office of Fusion Energy Sciences (OFES) fusion program.

In 1996, the OFES program was moved from DOE's energy programs to the Office of Science, and the program was restructured to emphasis plasma science towards an energy goal (hence the name). Since 1996, OFES funding and current guidance for funding has been flat except for an increase in FY00 for fusion, including 15\% for HIF, which was followed by a $10 \%$ cut for HIF for FY01. These budget constraints have forced us to postpone accelerator technology development for the Integrated Research Experiment (IRE), a \$200-300 M induction linac to validate driver cost scaling and integrated beam-chamber-target experiments. In March 2001 at the Washington budget-planning meeting, OFES Director Anne Davies asked the HIFVNL Director to clarify scientific results achievable in the HIF program under expected flat budgets for the next several years (and assuming no allowances for inflation). Beam science should be emphasized; accelerator technology development would be limited to that which would enhance existing science experiments. Although technology development for the IRE would be delayed under flat budgets, the beam science and technology enabling current experiments nonetheless still provide an important part of the basis for a future IRE.

In April 5-6 at an HIF-VNL Offsite retreat, the HIF-VNL Director asked the HIF task leaders, lead scientists, engineers and resource manager to complete a comprehensive 3-year research plan for the tasks we had proposed to DOE-Office of Fusion Energy Science (OFES) in our March Field Work Proposals (FWPs) for FY02/03. At that time the level of detailed planning was not sufficient to give confidence in the scheduled completion of all tasks for the flat guidance budget for FY02/03. The purpose of the three-year plan was to determine realistic schedules to achieve the proposed tasks. This report is an executive summary of the major results of six months of intensive reviews and planning, which has rebaselined and sequenced the FWP tasks over the next three years (in some tasks by four years). Our goal is to make most efficient use of available funds, while maximizing overall scientific progress (peer-reviewable scientific results) each year. In formulating this plan, we have made use of the results of four major VNL meetings during the past six months:

1. The offsite retreat April 5-6 improved our organization, meeting skills and teamwork. A vision for a next-step integrated beam experiment (IBX) designed to test integrated beam physics from source to target, but at much more modest scale and cost than an IRE, was proposed by the Director to guide the current research towards that next-step. 
2. The HIF-wide science workshop identified and prioritized the most important scientific issues facing the program. This 2-day workshop involved all scientists working within and with the HIF-VNL.

3. The internal review (with experts outside the VNL) of a first-draft 3-yr program plan, including experiments, theory and modeling, diagnostics, and cost, staffing and schedule requirements.

4. The second offsite retreat October 9-10 on the IBX defined a range of possible scientific capabilities and parameters for IBX, which will become part of a revised long range HIF development plan to be reviewed later in FY02.

A short description of the four major tasks areas of HIF-VNL research is given in the next section. The task areas are: High Current Experiment, Final Focus/Chamber Transport, Source/Injector/Low Energy Beam Transport (LEBT), and Theory/Simulation. As a result of the internal review, more detailed reviews of the designs, costs and schedules for some of the tasks have been completed, which will provide more precision in the scheduled completion dates of tasks. The process for the ongoing engineering reviews and governance for the future management of tasks is described in section 3. A description of the major milestones and scientific deliverables for flat guidance budgets are given in section 4. Section 5 describes needs for enabling technology development for future experiments that require incremental funding.

\section{Heavy-Ion Fusion Virtual National Laboratory Research}

\section{High current beam experiments in transport, sources/injection, and focusing}

The overall goal of HIF, to provide the comprehensive scientific knowledge base for IFE driven by high-brightness heavy ion beams, is consistent with the goal of the restructured Fusion Energy Sciences program, namely, to provide the scientific knowledge for fusion energy. The four top-level scientific issues for HIF are

1. What physics determines beam brightness in heavy-ion sources and low energy transport?

2. What dynamical phenomena affect the quality of space-charge-dominated beams undergoing transport and acceleration?

3. What role do non-linear processes and beam-plasma interactions play in beam propagation through the chamber and focusing onto a target?

4. How can we best apply and improve computational and analytical modeling to provide the needed support for experiments, exploration of issues, and planning for the future?

The HIF VNL is organized around four task groups to address these four scientific issues with separate experiments through FY02 and 03 that concentrate on specific issues affecting beam brightness. Particle-in-cell simulations and theory are in good agreement with past low current (few $\mathrm{mA}$ level) beam experiments studying transport, merging, acceleration and final focus. In those experiments, the dimensionless beam perveances (defined in the next paragraph) were similar to those in a driver. The program is now pursuing higher current experiments $(100 \mathrm{~mA}$ to $1 \mathrm{~A})$ : high brightness merging beamlet sources, transport at $\sim 2 \mathrm{MeV}$, and ballistic focusing with plasma neutralization. Improved simulation tools now enable modeling these experiments with realistic (not just idealized) beam particle distributions. Higher beam space-charge potentials associated with these higher-current experiments will allow us to study the effects of ionization of residual gas by the beam, trapping in the beam of stray electrons, and the dynamical effects on the beam of these two processes. We plan to collaborate in this area of common importance to the high energy physics community. A key strategic goal in 
theory/simulation is an integrated and detailed source-to-target simulation capability. We plan significant progress in this area over the next two years.

The above HIF-VNL program tasks are directly in support of the 5-year objective 6.1 identified in DOE's Integrated Program Planning Activity (IPPA), namely:

"Perform single-beam, high-current experiments to validate ion production, acceleration, and transport in a driver-relevant regime (line charge density 100 times greater than in present (2000) experiments".

Over the past several years the HIF program completed a series of low-current $(100 \mu \mathrm{A}$ to a few $\mathrm{mA}$ ) beam experiments in transport (SBTE, MBE-4, beam combiner experiment) and focusing (Scaled Final Focus Experiment) in which the beam kinetic energy and current were scaled to provide similar beam perveance as in the appropriate regions of a driver. Perveance is the dimensionless ratio of beam space-charge potential over ion kinetic energy, or the inverse square of the number of beam diameters a beam would propagate before doubling in size without external focusing fields applied. This parameter, Q, typically begins around $10^{-3}$ in the low energy end of the driver, decreases under acceleration to $\sim 10^{-5}$ to $10^{-6}$ at the high energy end, and then increases again to $10^{-5}$ to $10^{-3}$ as the beam is longitudinally compressed by a factor of 10 or more before final focus. Vacuum ballistic focusing in the target chamber would be feasible if the final beam perveance were $10^{-5}$ or less. Lower energy beams with final beam perveance of $10^{-4}$ to $10^{-3}$ can reduce accelerator cost but require some level of plasma neutralization of the beam space charge in the chamber. The past scaled beam experiments matched the predictions of ideal beam models, in which the effects of electrons, gas, and atomic physics could be neglected because the beam current, line-charge density and space charge potential were very low. The beam diameter was also typically small enough compared to the aperture of the focusing quadrupoles that the space-charge and applied focusing fields were sufficiently linear to preserve the normalized beam emittance. Under linear forces, Liouville's Theorem predicts conservation of beam phase space, as characterized by the normalized emittance. The normalized beam emittance for beams that are not expanding or contracting can be defined as the beam radius $a_{b}$ times the ratio of rms transverse ion velocity to the speed of light: $\varepsilon_{n x, y} \sim a_{b} \cdot\left(<T_{i \perp}>/ M_{i}\right)^{1 / 2} / c$, where the transverse ion temperature is $T_{i \perp}$ and $M_{i}$ the ion mass. Simulations show that when the beam space-charge and periodic external focusing forces are well-balanced so as to allow a periodic modulation of the beam envelope, but otherwise avoid sudden changes in the beam radius (good beam "matching"), then the normalized emittance, and the associated normalized beam brightness $\boldsymbol{\mathcal { B }}_{\mathrm{n}}=\mathrm{I} /\left(\varepsilon_{\mathrm{nx}, \mathrm{y}}\right)^{2}$, where $\mathrm{I}$ is the beam current, are nearly constant for a beam transported under linear focusing forces. For an accelerating beam bunch, and one undergoing longitudinal bunch compression, $\boldsymbol{B}_{\mathrm{n}} \Delta \mathrm{t}$ is conserved, where $\Delta \mathrm{t}$ is the bunch pulse duration. Nonideal effects, such as misalignment of the focusing magnets or field-strength errors can cause beam "mismatches", resulting in a small fraction of the beam electrostatic energy being converted into ion thermal heating, and resulting in degraded focusing. These effects are calculable and can be kept small with well-matched beamlines.

Having confirmed theoretical predictions of ideal beam dynamics with scaled experiments, we look next to experiments in FY02-03 at higher beam currents and at larger ratios of beam radius to focusing quadrupole radius, where the focusing fields develop nonlinear components, to explore limits that may be set by non-ideal physics in more driver-like beam regimes. At given beam perveance, as the beam current and ion kinetic energy both increase to driver-like levels, the absolute beam line-charge density $\lambda$ and space charge potential $\Delta \phi$ both increase, potentially drawing into the beam more electrons arising from beam halo ions 
intersecting the wall, and from direct beam-gas collisions. In the coming higher current experiments, we wish to explore the degree to which beam halos develop and may cause unwanted electrons and gas to perturb the desired balance of space charge and external focusing forces of a transported beam. We also need to explore the degree of achievable reductions of beam space charge in a focusing experiment where the beam entrains electrons from controlled chamber plasma. In this sense, the program is transitioning from low current "ideal" beam physics, to higher current "non-ideal" beam physics. The non-ideal effects we wish to study are expected to be important for HIF drivers, because they would determine how compact the transport channels could be designed, and how much lower ion kinetic energies could still be focused onto a target.

Over the next two years, the HIF-VNL plans a series of separate experiments in the areas of High Current Beam Transport (HCX), Sources/Injection/Low Energy Beam Transport (LEBT), and Final Focus/Chamber Transport (including NTX), all of which use currents in the range of $0.1-1 \mathrm{~A}$, where the beam potential exceeds a kilovolt. Mixed teams of staff from the three VNL labs will carry out these experiments. The total planned procurements (non-staff costs) to support these three experimental areas and supporting technology development is approximately $\$ 3 \mathrm{M} /$ year in the $\mathrm{VNL}$, which will diminish by $\sim 500 \mathrm{~K} / \mathrm{yr}$ due to inflation each year if guidance budgets remain flat.

\section{High Current Transport.}

This area includes a series of experiments (HCX- Figure 1) and supporting simulations on high current beam transport. These will use an existing ESQ injector and transport the beam $(1.8 \mathrm{MeV}, 0.6 \mathrm{~A})$ through a series of electric and magnetic quadrupoles to address a variety of issues, but primarily:

1. Alignment, beam sensing and steering: Misalignments cause oscillations in the beam centroid with a period characterized by $\sigma_{0}$, the undepressed particle phase advance. The oscillations bring the beam-edge closer to the physical aperture, resulting in larger nonlinear forces due to applied field errors and image charges. The frequency of needed steering corrections for tolerable centroid errors is important.

2. Radial extent of a beam halo resulting from, e.g., envelope mismatch and beam distribution non-linearities, and how to measure tenuous halo outside of the core beam. This issue is important because acceptable beam halo loss, and not emittance growth, may set the maximum beam fill factor.

3. Gas generated by lost ions impinging on the transport lattice aperture, and the influence of secondary electrons on beam dynamics.

For well-matched beams, simulations indicate that emittance growth should be small in these experiments, so we expect to be able to probe non-ideal effects limiting transport. These transport experiments will help us determine how compact the transport channels can be in the IBX, IRE, and future drivers, and therefore what maximum average beam current density can be transported through induction cores. In addition we expect to learn what combination of pulse lengths, vacuum requirements and wall conditioning may be required to manage the effects of secondary electrons, ions and atoms coming into the transport channel. Effects due to secondary 
electron production are expected to manifest themselves uniquely in magnetic quadrupoles, and may limit the beam-filling factor.

The envelope and collective modes that seed halo formation have rapid frequency oscillations that scale with the ion plasma frequency. Thus, it is essential to have an

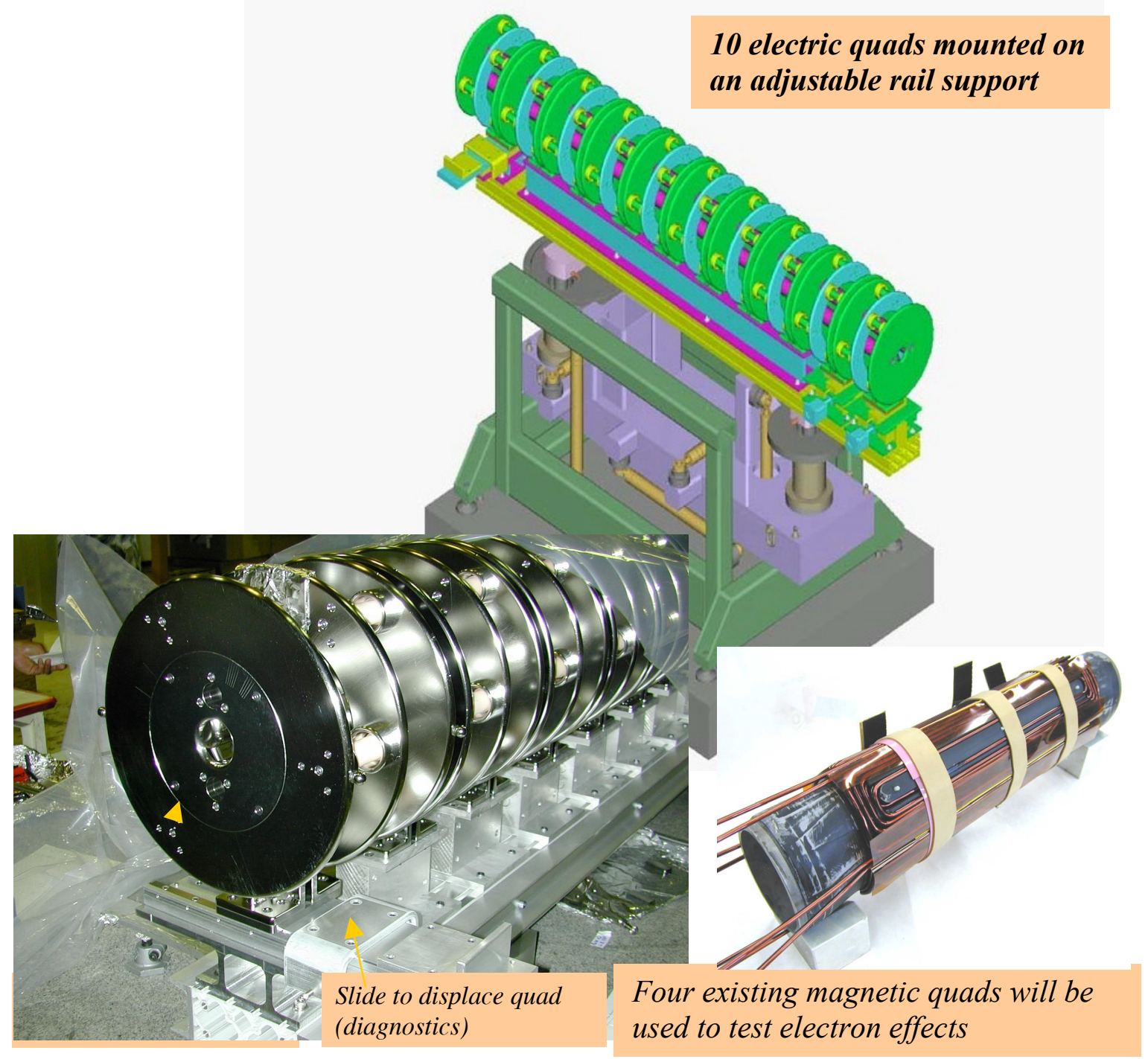

Fig. 1: The High Current Experiment (HCX). Operation begins January 2002.

experimental lattice length long enough for the beam to undergo several ion plasma oscillations. Flat budgets will allow us to construct a lattice of 30-40 electrostatic quadrupoles (4.5-6 plasma oscillations) followed by four pulsed magnetic quadrupoles. Clearly, an experiment with more (i.e., at least 40) magnetic quadrupoles to allow a sensitive probing of the dynamic aperture and limiting processes is highly desirable. This was planned for later phases of the HCX (under better budget conditions). However, even if budgets remain flat, it is planned to carry out preliminary experiments using 4-8 quadrupole prototypes from the room temperature (pulsed) and superconducting magnet $R \& D$ program. 
Magnet development for transport and acceleration experiments:

Present superconducting transport lattice designs center on magnet/cryostat systems being developed by the VNL laboratory and external partners (MIT, Advanced Magnet Lab, LLNL and LBNL) suitable for a transport experiment using the HCX beam described above. The present designs have a period of $2 \mathrm{~L}=45 \mathrm{~cm}$, a quadrupole occupancy of $\eta=0.5$, and superconducting wire at a radius of $r_{w}=3.5 \mathrm{~cm}\left(\mathrm{r}_{\mathrm{w}}>\mathrm{R}\right)$ with a peak field in the conductors of 67 Tesla. Prototypes of two different designs have been successfully tested in FY01. We will select a baseline design for further optimization and characterization of field quality in FY02. We also plan in FY02 to develop a unit transport cell (Figure 2) consisting of two quadrupoles sharing a common dewar, in a cryostat configuration compatible with acceleration gaps on both ends. This completes the magnet R\&D for single beam transport experiments. Unless more funding becomes available for FY03 and beyond, further SC magnet development cannot be carried out for multi-beam transport. Through the upcoming SBIR grant solicitation cycle, we hope to see the funding of the design and production of a prototype multiple beam array of superconducting quadrupoles, including the array cryostat, and cryogenic leads in a compact design that is compatible with induction acceleration modules.

As a possible lower-cost alternative to the superconducting magnets, we will design a prototype compact, high-field pulsed magnet capable of transporting the HCX beam. When more funding becomes available, we will build and test prototypes, and add them to the HCX transport lattice.

Fig. 2: An induction linac section showing three unit transport cells consisting of two superconducting quadrupoles per dewar, with an induction core between two of the transport cells. This design is one option considered for the IBX.

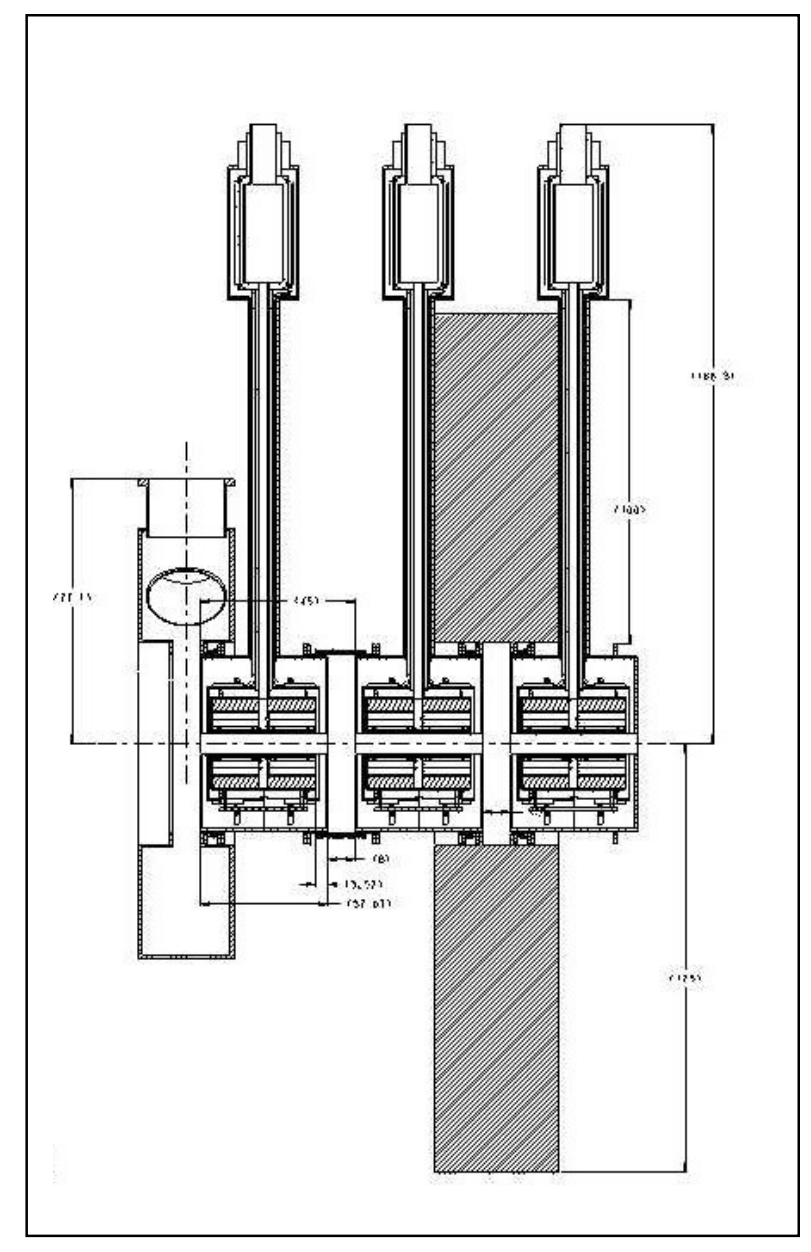


$\underline{\text { Longitudinal beam control and acceleration }}$

We will add two induction modules to HCX to study longitudinal control of the ends of the ion beam bunches and acceleration issues. In FY03, an induction module (from a phase 2 SBIR) will be installed on HCX, which will apply correction waveforms to the head and tail of the beam to correct for space-charge effects and regulate the flat top of the beam energy. A prototype induction module will be installed on HCX in FY04 to be used in later experiments to apply various acceleration waveforms to the beam to study acceleration issues and velocity tilt, and possible effects of the acceleration gap on electron physics. Also we will continue evaluation of available alloys for IBX induction cores.

All equipment for the first HCX transport experiments with 10 electric quads has been constructed or is in final assembly. First beam experiments on HCX are expected to begin in January 2002.

\section{Final Focus/ Chamber Transport.}

This area includes experiments and supporting theory/simulations on longitudinal drift compression, final focus and ion optics for ballistic and pinched beams, and heavy-ion stripping. It also includes beam plasma interaction in the chamber, particularly, plasma neutralization of beam space charge in the primary approach using ballistic chamber focusing. Plans for the next three years include more heavy-ion stripping experiments on existing heavy-ion beam accelerators in the US, Germany and Japan, as well as a high current neutralized beam transport experiment (NTX-Figure 3) that uses an existing $400 \mathrm{kV}$ injector, and an ECR plasma source developed to control the chamber plasma parameters for the experiment. The five major scientific issues to be investigated in this area are:

1. The limit on drift compression ratios set by sensitivity to initial variations in beam linecharge density $\lambda(\mathrm{z})$, velocity, and final perveance and resulting chromatic aberrations in final focus due to random and coherent velocity variations in the beam energy distribution. We will carry out theoretical studies and simulations of longitudinal drift compression for future experiments like IBX, IRE and drivers

2. Obtaining improved data for accurate predictions of gas pressure limits for tolerable beam stripping.

3. The limits to the focusable beam perveance. We plan to explore the range of $10^{-5}$ to $10^{-3}$ at 100-1000 x greater line-charge densities than in the scaled focus experiment.

4. The limits of beam space charge neutralization as functions of plasma to beam density ratios, with various axial plasma gradients and locations outside and inside the chamber regions.

5. The evolution and stability limits of intense ion beams in ballistic and pinched propagation modes.

Studies of the various limits to practical drift compression ratios will help us define the critical experiments and measurements needed on drift compression in the IBX, and the relevance of those experiments to the IRE and drivers. Improved data on beam stripping will improve designs for vacuum pumping of chamber vapor streaming up the beam lines in the IRE and drivers, and will lead to improved understanding of the net effect of competition between stripping and electron neutralization in the chamber itself. Understanding the limits on focusable beam perveance will be a key factor in the determination of the desired number of beams in the IRE to 
achieve desired peak beam intensities on targets. Understanding limits on plasma neutralization of beam space charge in the chamber will be a key factor in determining the minimum focal spot sizes on target as a function of ion kinetic energy in the IBX, IRE, and drivers. A broad study of several propagation modes could enlarge the operating parameter window with potentials for large payoffs in relaxed driver and chamber requirements.

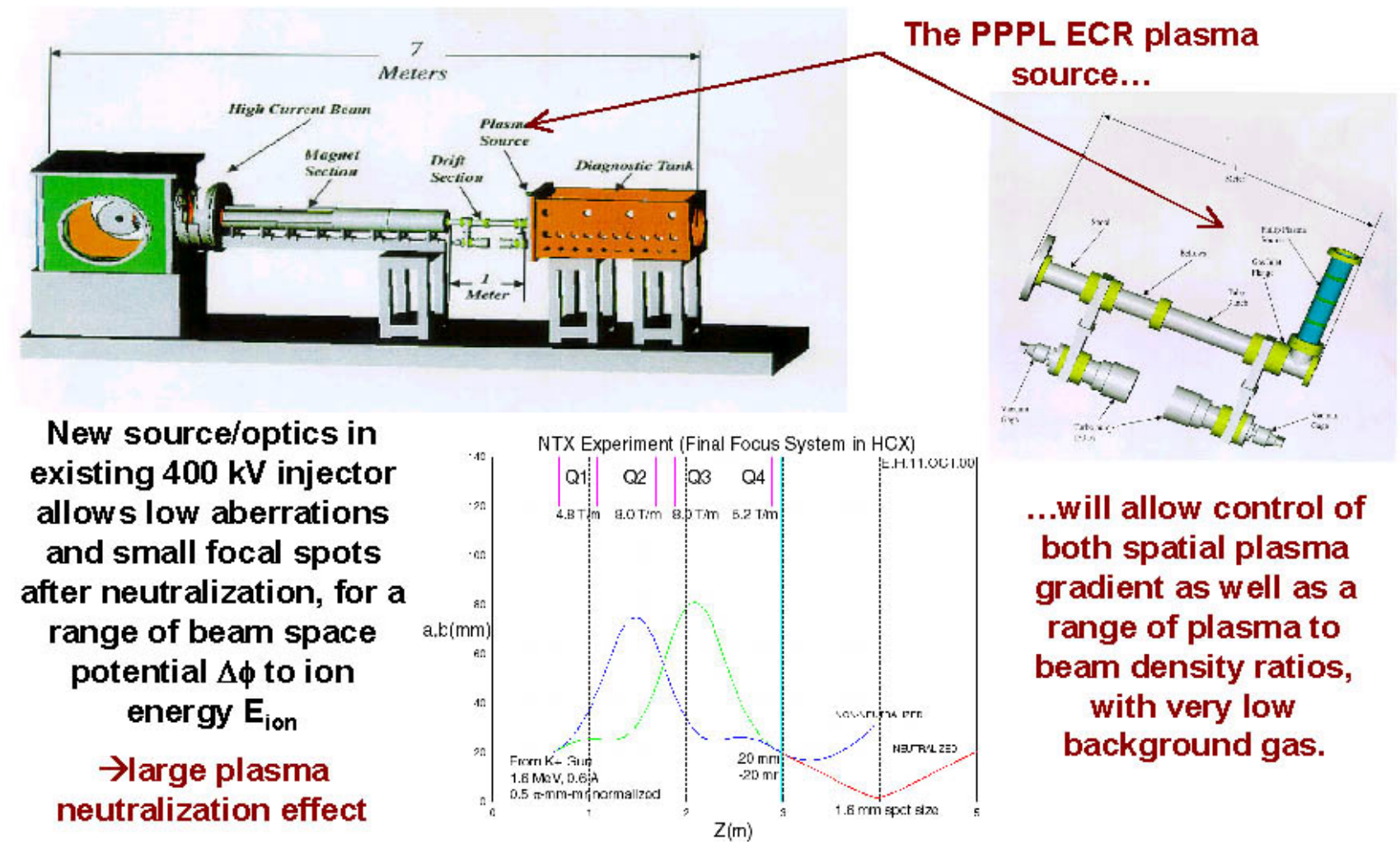

Fig. 3 The Neutralized Transport Experiment (NTX) First NTX beam experiments expected to begin FY03

\section{Sources/Injection/LEBT.}

This area includes experiments, theory and simulation for the development and improvements to injectors needed in the transport and focusing experiments, and for future experiments such as the IBX and IRE. The facility will enable research not only on ion sources and injectors, but also contribute to understanding multiple beam physics, long-pulse beam-gas interaction, and voltage gradient limits. We anticipate that experiments with high current density sources and injection will lead to brighter, more durable, and more compact injectors for future HIF experiments. A major research focus will be a better understanding of issues that dictate the choice of large-area sources versus compact merged- beam sources wherein a large beam is formed by merging many small beamlets.

The traditional way of producing high current beams for HIF is a large diameter surface ionization source. The current density is limited by the injector's ability to focus a large current beam (due to the beam's space charge expansion force). However, large diameter beams have an 
inherent spherical aberration at the beam edge. This aberration is difficult to calculate with precision and to correct. The brightness will improve if the beam edge can be "cleaned up" by passing through an aperture to eliminate the aberrated edge. Furthermore the aperturing method could be used to vary beam current allowing additional experiments to be performed. However, aperturing of intense ion beams to increase brightness is not well understood as electrons produced by ions impinging on the orifice may degrade beam quality. Therefore, we intend investigation of aperturing to better comprehend beam behavior in these areas. During these experiments we will also gain some insight into long pulse, beam-gas interaction effects.

A novel concept is proposed to produce compact high current injectors. Merging an array of mini-beamlets into one high current beam is expected to eliminate the aberrated edge effects of conventional large diameter beam optics. This concept builds on the experience of neutral beam sources developed earlier for MFE. The array of mini-beamlets must be extracted from a high current density ion source with a sufficiently high transparency through the accelerator grids in order to achieve high brightness.

Source and Injector key scientific issues are to:

1. Understand the average current density achievable for both large diameter, and merged-beamlet sources.

2. Understand the causes of emittance growth for both geometries.

3. Investigate limits for high-gradient insulators and vacuum gap voltage breakdown.

4. Determine the feasibility of extracting and injecting beams with adequately short beam current rise times while minimizing beam mismatch in low energy transport.

5. Investigate feasibility of increasing brightness by aperturing beams at the end of the injector to remove aberrated portions at the beam edge.

Over the next 6-7 years STS-500, the newly constructed injector beam test facility using a $500 \mathrm{kV}, 20 \mu \mathrm{s} \mathrm{HV} \mathrm{supply} \mathrm{(Fig.} \mathrm{4)} \mathrm{will} \mathrm{study:}$

1. A variety of candidate ion sources of the two injection beam geometries. If budgets remain flat, the priority is to evaluate surface ionization sources for both geometries, and then plasma sources.

2. Multiple beam electrostatic interactions, and electron effects.

3. Beam-gas interaction physics including charge exchange and long pulse outgassing.

4. Advanced technologies for very high voltage gradients in insulator stacks and vacuum gaps.

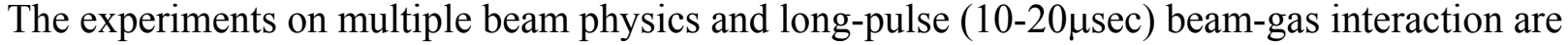
important to the design of future multiple beam induction linacs such as the IRE and drivers, since beam-beam repulsion within acceleration gaps must be accounted for in beam centering within each transport channel. The advanced high-gradient tests will potentially reduce IRE and driver accelerator length, and therefore a cost by a factor of 2 to 3 .

High voltage gradient insulators and vacuum gaps will be investigated in the first tests of the STS-500. Initial testing is to confirm voltage-holding capacity of the insulator structure. Vacuum gap breakdown will be studied with various surface cleaning and processing techniques. A follow-on set of experiments will determine insulator performance in the presence of an ion beam. Initial plasma source experiments are to be conducted on the STS-100 (an existing test stand within the Source Test Laboratory at LLNL with 100keV capability). 

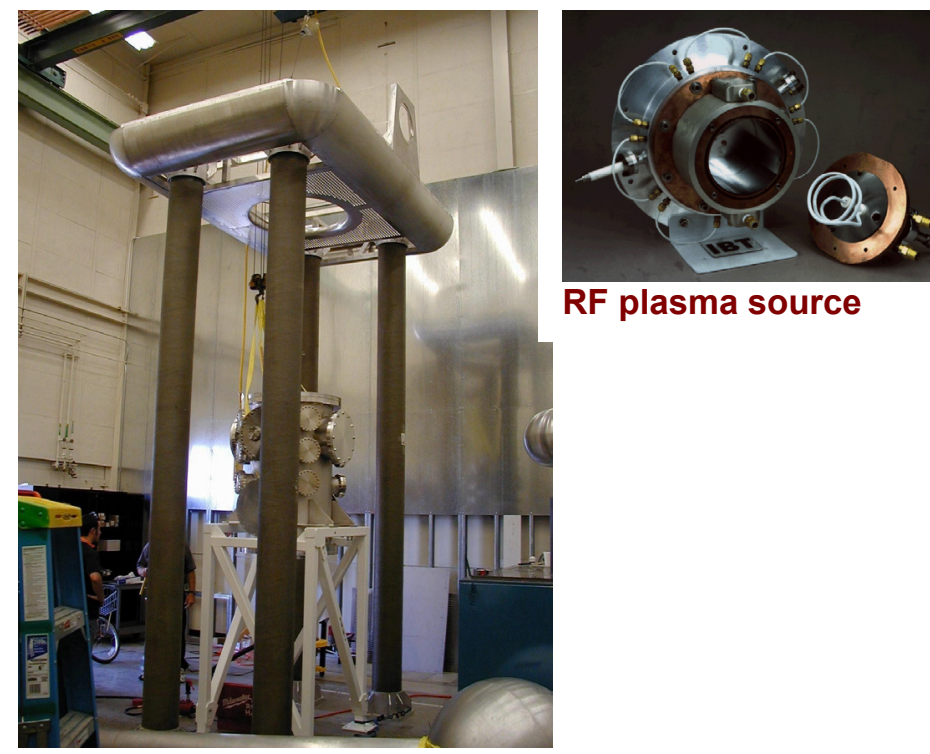

RF plasma source

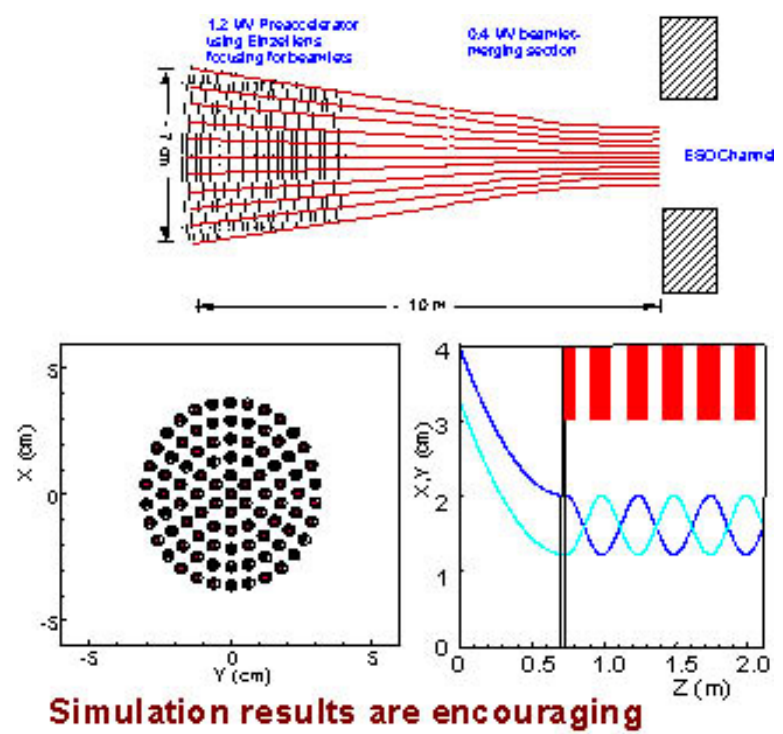

Fig. 4: STS-500 (1), a versatile $500 \mathrm{kV}$ test stand for source and multiple-beam physics experiments is, nearing completion at LLNL. Plasma source testing will proceed on STS100 in FY02, and design efforts for the first multiple beam source for STS500 are expected to be completed in FY02.

\section{Theory and Simulation}

A central goal of the HIF program is to provide comprehensive simulation and modeling of ion beams, from the source to the target. The simulation and theory work guides our research, supports modeling and simulation of the beam experiments, and develops the improved computational tools needed for future experiments. The HIF theory program has made important progress in understanding ion beams with orders of magnitude more charge than in traditional accelerators, but important issues remain. This effort is divided into roughly $2 / 3$ simulations and $1 / 3$ analytical theory, in three major areas:

1. Analytical and numerical studies contributing to the design and interpretation of experiments, including HCX, NTX, and injector [slightly over half the overall theory and modeling effort].

2. Development of the beam physics knowledge base, and the scientific basis for future experiments. We seek to ensure that no key conceptual issues remain unexplored. We are distilling our understanding into improved scaling laws, models, and algorithms, and are incorporating these elements into our beam systems code. We are also laying the groundwork for future integrated beam experiments (IBX), as well as for an IRE and a full-scale driver. The emphasis is on synthesis of our beam physics knowledge into realizable concepts, and on the critical assessment of those concepts [just under a quarter of the overall effort]. 
3. Development of computational tools. Our ultimate goal is integrated source-to-target simulation capability that we can exploit so as to proceed confidently with our experimental program [just over a quarter of the overall effort].

The HIF program has developed unique simulation tools to explore the relevant physics areas. Our codes are characterized by their methods and regimes of applicability:

1. Follow particles (plasma particle-in-cell method):

-WARP (driver): 3-D (or r,z or x,y) electrostatic (ES), detailed lattice. This code offers a detailed beamline description but is suitable for studies of propagation over long distances. It uses novel "cut-cell" boundaries to allow subgrid-scale resolution of internal structures. It has been benchmarked versus HIF experiments, and others. The name derives from its use of "warped" coordinates to model bent beam lines.

-LSP (chamber \& driver): 3-D or (r,z) implicit (or explicit) electromagnetic (EM) or electrostatic, hybrid (kinetic/fluid). This code is optimized for the study of systems with a wide range of scales and high-density plasmas. It has been benchmarked on a variety of applications inside and outside HIF. The name is an acronym for "large scale plasmas."

\section{Follow particles and perturbation $(\delta f)$ to the beam distribution function:}

-BEST (chamber \& driver): 3-D EM, Darwin, or ES. This code uses a nonlinear-perturbative formulation to minimize the discrete-particle noise. As such, it is especially suitable for detailed studies of modes on a beam, where the structure of the modes is of significance. It is serving as a testbed for the development of a novel magneto-inductive (Darwin) model. It has been benchmarked versus experiments on the LANL PSR storage ring. The name is an acronym for "beam equilibrium, stability, and transport."

\section{Evolve moments of distribution function:}

- CIRCE \& HERMES (driver): transverse moments, longitudinal Lagrangian fluid. These models exist within the WARP framework (CIRCE also runs stand-alone), and couple transverse "envelope equations" at multiple "slices" of a long beam via Lagrangian fluid equations. The models differ in their internal representations; both are useful for scoping and synthesis, e.g. for drift-compression and pulse shaping.

Other HIF codes are worthy of mention. The BIC and BPIC particle-in-cell codes are also used for chamber transport studies. BIC was our first such tool, and offers an axisymmetric model in converging geometry. BPIC offers both axisymmetric and 3-D models, and includes optimized outgoing-wave EM boundary conditions (a development which should have impact in many fields) as well as a new method of "advecting away" accumulated numerical errors. It is our plan to introduce the best features of these other codes into LSP, which we envision as our principal entrance-to-target chamber simulation tool.

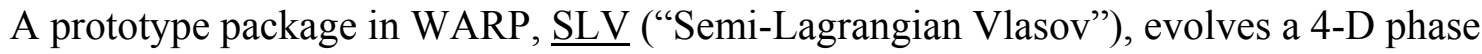
space density on a grid. This description is especially suitable when low-density regions of the phase space must be accurately represented, as in studies of beam halo. At present, the package handles only uniform focusing, but quadrupole focusing should be treatable after development along well-defined lines; however, that work is not included in the flat-budget case. 
Some examples of the application of some of these tools appear in Figure 5 below.

Ion beams are collisionless, and features of the particle distribution persist for long times, so initialization of a beam at mid-system with an idealized particle distribution introduces inaccuracies; thus, it will be important to develop, and to extensively use, an integrated and detailed "source-to-target" HIF beam simulation capability. Development of such a comprehensive source-to-target simulation capability is an important strategic direction for the HIF program. Testing and validating this integrated modeling capability will be a main objective of the IBX. In our proposed scenario for integrated modeling, the beam is simulated from the source through the final focusing optic using WARP3d, and the particle and field data are then transferred into LSP where the simulation is carried through the chamber plasma environment to the fusion target. At that point the particle data is used to generate "ray" information for the ion beam source in the radiation-hydrodynamics target design code (currently LASNEX, but ultimately a 3-D code). Figure 6 illustrates how the various codes connect to sections of the accelerator and focusing systems, in this proposed strategy for integrated modeling.

LSP can also be used to study electron effects in the driver, especially sources and trapping in the beam. For this to be accurate, it is necessary to understand beam halos quantitatively, and for this the marker-following capabilities of BEST, WARP with an extralarge number of particles, and/or extensions of the Vlasov solver SLV can be employed in coupled side calculations, beginning with the beam state as obtained in the "main sequence" of runs. BEST is already being used to study collective instabilities in detail, including two-stream instabilities involving the interaction of beam ions with background electrons. 

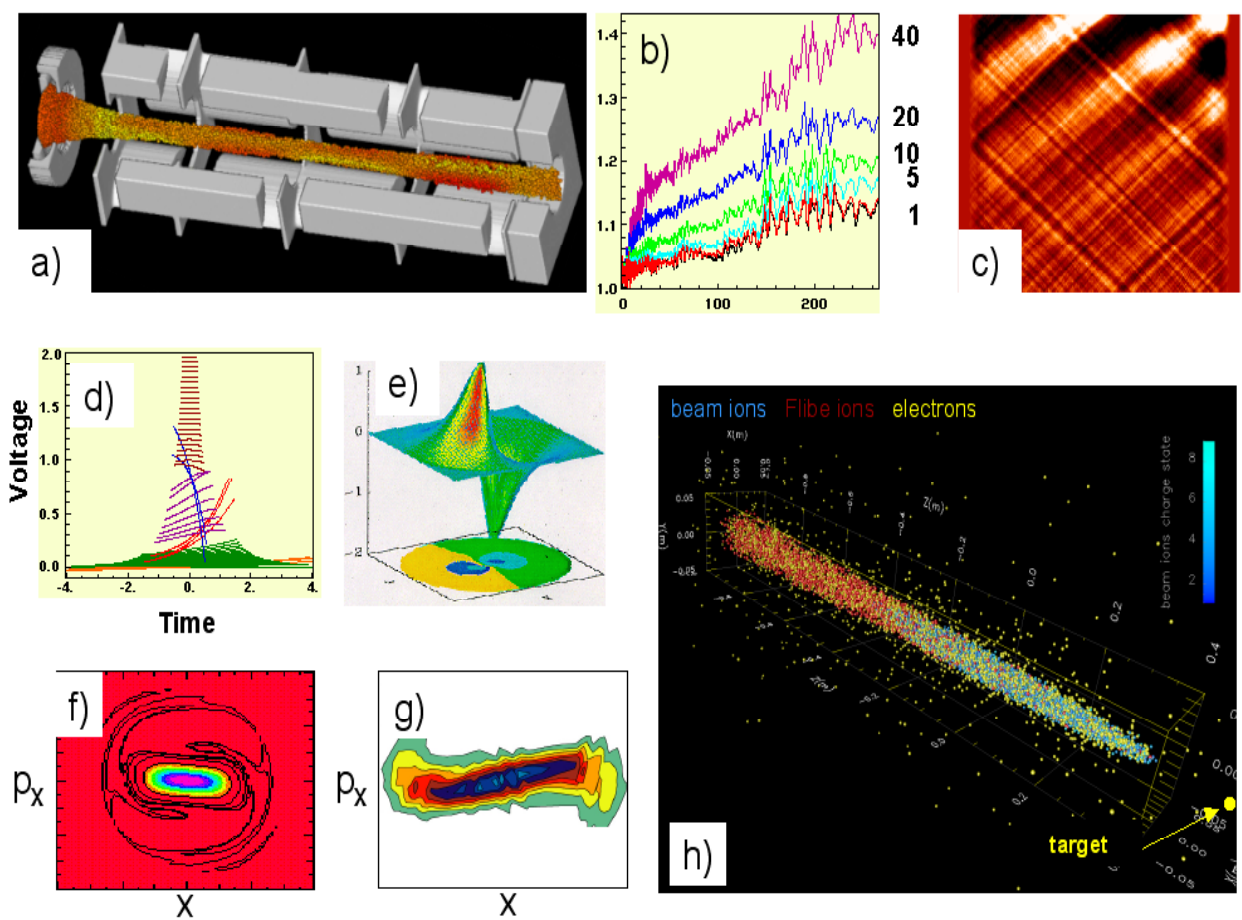

Fig. 5. Representative output from HIF beam simulations: (a) WARP3d simulation of spacecharge-limited emission and acceleration in a 3-D structure, including subgrid-scale placement of conductor boundaries; (b) WARPxy study of beam emittance versus time in an imperfectlyaligned beamline, for five different intervals between applications of steering; (c) WARP3d study of unstable longitudinal waves (space-time diagram; color is perturbed density); (d) accelerating waveforms for a possible future accelerator, for use in WARP3d simulations; (e) BEST simulation of unstable electron-ion two-stream mode; (f) semi-Lagrangian Vlasov simulation of beam halo generation due to anharmonic focusing fields, using prototype model in WARP-SLV; (g) distorted beam phase space in final focusing, as simulated using WARPxy; (h) BPIC simulation of beam transit through fusion chamber environment and onto the target.

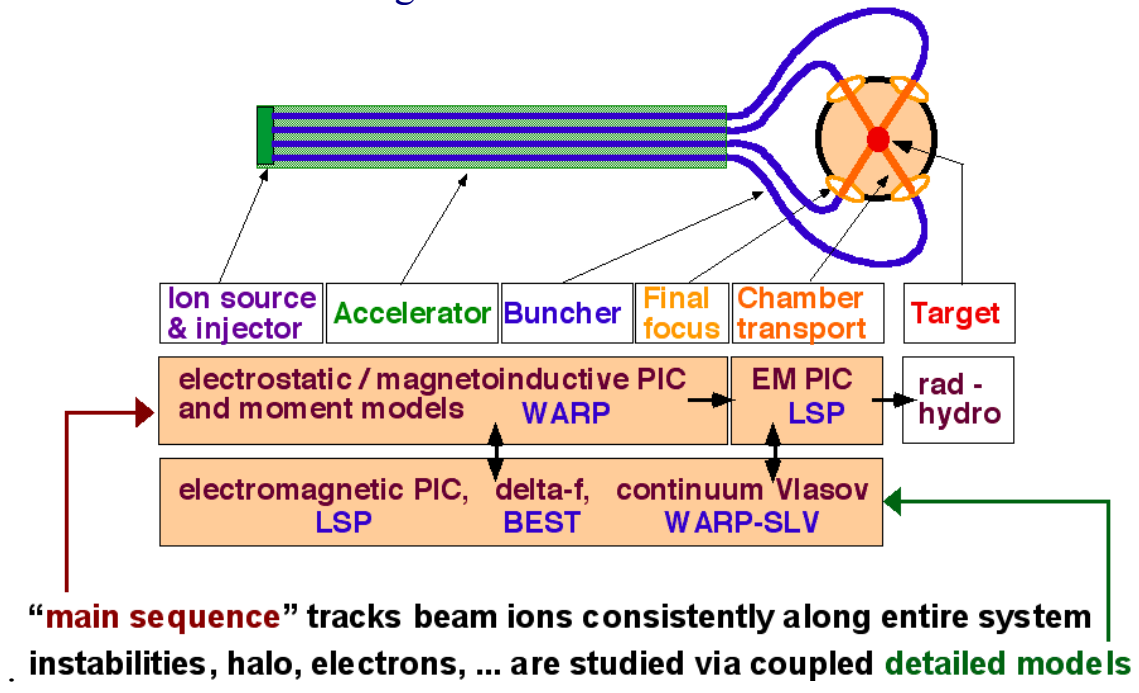

Fig. 6. Schematic of a heavy-ion linac, showing domains of applicability of the various classes of simulation codes to be employed in simulations of beams from source to target. 


\section{Budgets, Milestones and Scientific Deliverables}

Table 1 below gives the allocations (in as spent \$K dollars each year) for the four task areas from the total $\$ 10.6 \mathrm{M} / \mathrm{yr}$ of tri-lab funding for the HIF-VNL. The priorities which determined these allocations are listed in Table 2. There is $\sim 200 \$ \mathrm{k}$ of additional support for VNL activities at SNL and NRL not included in this VNL budget plan, which is separately funded by OFES.

Table 1: Task Area Allocations (\$K, inc. base staff + operating) by task area next 3 years.

\begin{tabular}{|l|c|c|c|}
\hline VNL-LBNL/LLNL/PPPL & FY02 & FY03** & FY04** \\
\hline HCX & $\mathbf{3 1 4 8}$ & $\mathbf{3 6 8 7}$ & $\mathbf{3 3 9 5}$ \\
\hline Final Focus/Chamber/NTX & $\mathbf{2 6 6 5}$ & $\mathbf{2 4 7 1}$ & $\mathbf{2 5 3 2}$ \\
\hline Sources/Injector/LEBT & $\mathbf{2 4 5 7}$ & $\mathbf{2 7 0 7}$ & $\mathbf{2 8 2 4}$ \\
\hline Theory/Simulation* & $\mathbf{1 6 0 8}$ & 1731 & $\mathbf{1 8 3 3}$ \\
\hline Management Reserve & $\mathbf{4 5 0}$ & $\mathbf{4 0 0}$ & $\mathbf{3 5 0}$ \\
\hline \multicolumn{1}{r|}{ Totals } & $\mathbf{1 0 3 2 8}$ & $\mathbf{1 0 9 9 6}$ & $\mathbf{1 0 9 3 4}$ \\
\hline
\end{tabular}

*Theory/Simulation outside of direct support included in above experiments

** "Flat budget planning" assumes restoration of $260 \mathrm{~K}$ of FY02 cuts compared to FY01, and $\$ 300 \mathrm{~K}$ redirection of non-HIFVNL IFE funding to support high priority HIF-VNL experiments. A breakdown of the above FY02 funding by institution is shown below (institutional funding splits for FY03/04 TBD)

\begin{tabular}{|r|c|c|c|}
\hline & \multicolumn{3}{|c|}{ FY02 \$k } \\
\hline VNL location & Operating & Equipment & Total \\
\hline LBNL & 5241 & 397 & 5638 \\
\hline LLNL & 3585 & 0 & 3585 \\
\hline PPPL & 1105 & 0 & 1105 \\
\hline Total & 9931 & 397 & $\mathbf{1 0 3 2 8}$ \\
\hline
\end{tabular}

Table 2: HIF-VNL priorities, which guides allocation of VNL funds among tasks

(1) $10 \mathrm{EQ}+4$ MQ HCX transport and electron physics experiments in FY02. Science workshop issues: diagnostics, alignment, steering, electron physics.

(2) NTX construction in FY02 for ballistic neutralized focus experiments beginning FY03. Science workshop issues: FF perveance limits, plasma neutralization.

(3) Procure more half-lattice periods in FY03 for HCX experiments. Science workshop issues: Halo and emittance growth versus fill factor for longer transport, more plasma oscillations.

(4) Scaled-beamlet accelerator structure design, build and voltage test in FY02 for $500 \mathrm{kV}$ experiments beginning FY03. Science Workshop issues: feasibility of multi-beamlet approach versus large beams, to improve future sources.

(5) HCX longitudinal beam control experiments. Science workshop issues: longitudinal head-tail control physics.

(6) Superconducting magnet development. Science workshop issues: low cost, compactness and field quality. 
Table 3: Milestones and Scientific Deliverables by Task Area and Year

\begin{tabular}{|c|c|c|c|}
\hline Task Area & FY02 & FY03 & FY04 \\
\hline $\mathrm{HCX}$ & $\begin{array}{l}\text { Diagnosis of } \\
\text { alignment/mismatch } \\
\text { effects, fill factor, } \\
\text { electron physics with } 10 \\
\text { EQ's. }\end{array}$ & $\begin{array}{l}\text { Electron physics with } 4 \\
\text { MQ's. Extend transport } \\
\text { experiments to } 4.5-6 \\
\text { plasma periods. }\end{array}$ & $\begin{array}{l}\text { Study halo growth. } \\
\text { Bunch end control. } \\
\text { Design/build induction } \\
\text { module for acceleration } \\
\text { dynamics }\end{array}$ \\
\hline $\begin{array}{l}\text { Final Focus/Chamber } \\
\text { Transport/NTX }\end{array}$ & $\begin{array}{l}\text { Characterize of ion and } \\
\text { plasma sources to be } \\
\text { applied in NTX. }\end{array}$ & $\begin{array}{l}\text { Complete beam } \\
\text { aberrations study. } \\
\text { Preliminary results on } \\
\text { neutralization. }\end{array}$ & $\begin{array}{l}\text { Complete initial } \\
\text { neutralization } \\
\text { experiments. }\end{array}$ \\
\hline Source/Injector/LEBT & $\begin{array}{l}\text { Complete initial } \\
\text { optimization of rf-plasma } \\
\text { sources; Initial testing of } \\
\text { multi-layer high gradient } \\
\text { insulator without beam. }\end{array}$ & $\begin{array}{l}\text { Design and fabricate } \\
\text { multi-beamlet accel } \\
\text { structure; Complete } \\
\text { beam aperturing study; } \\
\text { Complete testing of } \\
\text { multi-layer insulator } \\
\text { with beams. }\end{array}$ & $\begin{array}{l}\text { Complete beam } \\
\text { brightness study, select } \\
\text { injector configuration } \\
\text { for future development; } \\
\text { Complete full-voltage } \\
\text { beamlet acceleration to } \\
500 \mathrm{kV} \text {; Begin } \\
\text { advanced source } \\
\text { prototype development. }\end{array}$ \\
\hline Theory/Simulation & $\begin{array}{l}\text { Improved spot size model } \\
\text { for systems code; } \\
\text { Simulation of near-target } \\
\text { pinch effects; Synthesis of } \\
\text { 4-D beam distribution } \\
\text { from measurements }\end{array}$ & $\begin{array}{l}\text { 3-D simulation from } \\
\text { linac to chamber; } \delta \mathrm{f} \\
\text { simulation of pressure } \\
\text { anisotropy-driven } \\
\text { modes; Transverse } \\
\text { multi-beam analysis }\end{array}$ & $\begin{array}{l}\text { Longitudinal non- } \\
\text { identical beam studies; } \\
\text { Simulation of electron } \\
\text { effects mitigation; } \\
\text { Studies of beam } \\
\text { overlap near target. }\end{array}$ \\
\hline
\end{tabular}

Short descriptions of the deliverables in this table are given on following pages. 


\section{$\underline{\text { Key HCX milestones and scientific deliverables }}$}

The objective of the HCX is to obtain data on how these processes limit transportable beam current and brightness:

- Non-linear applied fields and non-linear fields from the beam distribution,

- Alignment, beam sensing and control.

- Lost particles and halo formation.

- Interaction of the beam with secondary electrons, ions and atoms.

- Consequences of longitudinal phase space distribution on transverse dynamics.

The radial extent of the beam, or fill factor $\left(r_{\text {beam }} / r_{\text {pipe }}\right)$, will be varied parametrically to probe the deleterious effects, which can be enhanced at high fill factors (and currents) desirable for HIF.

\section{FY02}

Diagnosis of alignment/mismatch, fill factor with 10 EQs: Misalignments cause oscillations in the beam centroid with a period characterized by the betatron particle frequency. The oscillations bring the beam-edge closer to the physical aperture, resulting in larger nonlinear forces due to applied field errors and image charges. Limitations on sensing and controlling the beam centroid and envelope will be characterized in this early round of measurements.

\section{FY03}

Electron physics with 4 MQs: Secondary electrons present an issue of concern in magnetic focusing systems. Such systems lack the strong sweeping fields associated with electric focusing, increasing chances that electrons will be trapped in the multi-kV potential well of the ion-beam thereby leading to streaming instabilities, partial neutralization, and loss in beam control. These will be characterized with new diagnostics.

We will extend transport experiments to 4.5-6 plasma periods (30-40 EQs): The envelope and collective modes that seed halo formation have rapid frequency oscillations that scale with the ion plasma frequency. The extended transport lattice will be long enough to allow several plasma oscillation periods, which will be sufficient for studies of collective processes leading to halo production and emittance growth.

\section{FY04}

We will characterize halo growth and phase space evolution as a function of fill-factor: Beam distribution distortions and mismatches in phase-space can launch deleterious collective oscillations. Mismatches of the distribution to focusing structures lead to beam envelope oscillations that can pump large amplitude beam halo. Higher order distortions such as those resulting from space-charge nonuniformities may launch a spectrum of collective waves that complicate beam control and can lead to emittance growth. Measurements of envelope and collective modes with rapid frequency oscillations that scale with the ion plasma frequency will be interpreted with several simulations and analysis.

Bunch end control: an induction module (from a Phase-2 SBIR award) will be installed on HCX, which will apply waveforms to the head and tail of the beam to correct for spacecharge effects and regulate the flat top of the beam energy.

Induction modules for acceleration dynamics: A prototype induction module will be designed, fabricated and installed to apply various acceleration waveforms to the beam to study acceleration issues and velocity tilt. 


\section{$\underline{\text { Key Final Focus/Chamber Transport milestones and scientific deliverables }}$}

The NTX experiments are necessary to test theory and simulations of the limits on focusable beam perveance and limits on plasma neutralization of beam space charge in the chamber. This will improve our understanding of what determines the minimum focal spot sizes on target as a function of ion kinetic energy in the IBX, IRE, and drivers. Some key supporting theory/simulation milestones in the final/focus/chamber area are described in the following section on theory/simulation milestones.

\section{FY02}

The high perveance ion gun is needed to study beam aberration and neutralization effects. To provide a sensitive measure of the degree of neutralization, the beam must have low emittance, which we will seek to demonstrate.

The ECR plasma source with variable density and axial profile is an essential component of the neutralization experiment. It will be quantitatively characterized prior to installation into the NTX beamline.

The NTX beamline, including 4 pulsed quadrupole magnets, will be under construction in FY02.

\section{FY03}

Beam aberration experiments will be completed. These experiments will measure distortion of the phase space due to nonlinearities in magnetic quadrupoles as a function of beam perveance. This is one of the key experiments in determining the perveance limit.

Neutralization experiments are expected to require several iterations. The first test will be done in FY03.

\section{FY04}

Initial neutralization experiments will be completed under various conditions of plasma density and axial locations, as well as gas densities. Experimental results will be compared with simulations to quantitatively address beam neutralization effects. Additional neutralization experiments may be needed in FY05, depending on physics results.

Octupole corrections of beam aberrations, an essential measure for extending the operating space of the final focus system, will be studied in FY05. 


\section{Key Source/Injector/LEBT milestones and scientific deliverables}

The objective of ion source and injector research is to provide HIF experiments, and future drivers, with heavy ion beams that are both high current and high brightness. In simple words, high brightness means a good quality beam with minimal divergence. Therefore it is not surprising to find that the two conditions of high current and high brightness are difficult to achieve simultaneously. We have carried out preliminary theory and simulation studies to show that the final beam brightness of a merged array of mini-beamlets has the advantage of significantly reducing the injector size and cost, and the following scientific program addresses this.

\section{FY02}

High current density ion sources will be investigated in FY02. Our two main ion source approaches are miniature surface ionization sources with current density ( $\mathrm{K}^{+}$equivalent) at about $50 \mathrm{~mA} / \mathrm{cm}^{2}$, and rf-driven plasma sources at about $100 \mathrm{~mA} / \mathrm{cm}^{2}$.

One universal way to obtain high current is to elevate the current density by maximizing the acceleration voltage gradient. To improve this limitation, we will conduct tests on the newly developed multi-layer high gradient insulators, initially without the presence of ion beams in FY02.

\section{FY03}

Studying large diameter beams and aperturing experiments, done together with theory and simulation support, are expected to completed in FY03. We intend to complete the design of a mini-beamlet acceleration grid (full-size, designed to hold 1.6 MV) and fabricate a scaled version for $500 \mathrm{kV}$ tests. Although our first experiments in FY03 with mini-beamlets acceleration and merging will be done at a reduced voltage of $500 \mathrm{kV}$ (as limited by the test stand power supply), it will provide the physics validation of this concept. Both design work and data analysis will require significant amount of theory and simulation supports. We plan to test multi-layer high gradient insulators with the presence of ion beams in FY03.

\section{FY04}

In FY04, we will test the first 1/3 section of the grid assembly at full voltage gradient in order to demonstrate the current density and voltage holding capabilities. Together with the experimental results from large diameter beams, we plan to complete the beam brightness study and therefore make a selection between the two configurations for future injector development.

Adopting high-gradient insulator in the accelerator design will result in higher performance and reduction in size. 


\section{Key Theory and Simulation Milestones and Scientific Deliverables}

A central goal of the HIF program is to provide comprehensive simulation and modeling of ion beams, from the source to the target. The simulation and theory work guides our research, supports modeling and simulation of the beam experiments, and develops the improved computational tools needed for future experiments. Ion beams are collisionless, and features of the particle distribution persist for long times, so initialization of a beam at mid-system with an idealized particle distribution introduces inaccuracies; thus, it will be important to develop, and to extensively use, an integrated and detailed "source-to-target" HIF beam simulation capability. Development of such a comprehensive source-to-target simulation capability is an important strategic direction for the HIF program.

\section{FY02}

Improved spot-size model for systems code: We will develop an improved analytic model for the beam spot size on the fusion target, incorporating improvements in our understanding of intense-beam focusing optics, and allowing for the possibility of elliptical spots. We will validate the model using transverse (x,y) WARP-code simulations, revise it as necessary, and implement it in the HIF beam systems code.

Simulation of near-target pinch effects: We will implement more accurate boundary conditions, and models for beam and plasma stripping to multiple charge states, into the LSP code. We will use the improved code to develop an understanding of recently-discovered neartarget pinching in neutralized-ballistic chamber propagation. This is important because the effect can influence the focusability of a beam onto the target

Synthesis of 4-D beam distribution from measurements: We seek to launch realistic particle simulations based on initial conditions that are obtained from experimental measurements. However, present-day diagnostics yield only projections of the full multidimensional phase space, so the synthesis of the full distribution is an underdetermined problem. We are developing a Monte-Carlo method that adds physically-motivated constraints to complete the specification, and will report on this method and its initial use.

\section{FY03}

3-D simulation from linac to chamber: We will develop and examine consistent accelerator-to-chamber scenarios for both NTX and future integrated beam experiments. We will employ the WARP code to carry out 3-D particle simulations of beam drift compression and final focusing, so as to better understand these processes. Transverse and longitudinal effects are tightly coupled here, and so reduced descriptions are insufficient. This work will include studies of strong chromatic aberrations (dependencies of the focal spot on particle kinetic energy) and their control by pulsed focal elements.

$\delta$ fimulation of pressure anisotropy-driven modes: A beam in which the transverse beam temperature significantly exceeds the longitudinal temperature can undergo a collective instability, which drives the spreads closer to equality. The growth and saturation of this mode, and its detailed structure, are being studied using the nonlinear-perturbative $\delta f$ code BEST, and the findings will be described in a report. 
Transverse multi-beam analysis: Toward the high-energy end of a driver, the accelerating gaps represent the largest fraction of the machine "lattice," and it is here that interbeam electric and magnetic forces can be significant. We will develop an improved understanding of transverse multi-beam deflections, and will analyze advanced concepts for accelerating gaps that minimize such unwanted effects. We will begin the formulation of a multibeam simulation model for the gap architecture deemed most promising with respect to longitudinal and transverse equilibrium and stability.

\section{FY04}

Longitudinal non-identical beam studies: We will develop the first version of a novel new model in the WARP particle-in-cell code: a true multi-beam model wherein one beam is simulated in full detail, the other beams are modeled at a simplified level, and each beam can behave independently. The first studies of longitudinal wave propagation and stability in this new but driver-relevant regime will be carried out.

Simulation of electron effects mitigation: Electron cloud space-charge effects in beams transported by magnetic quadrupoles may degrade the emittance of the beam or lead to particle loss. We will improve our analysis and simulations of electron trapping in a multi-magnet system including accelerating gaps, will include the effects of mobile ions, will study a variety of mitigation schemes if needed, e.g., beam "halo" limiters and electron capture electrodes, and will report on this work.

Studies of beam overlap near target: We will develop a true multi-beam simulation capability in the LSP code, and will conduct the first simulations of transport in the neutralizedballistic regime that incorporate multi-beam overlap near the target. 


\section{Plan for managing and documenting directed and unscheduled variances to cost and schedule of HIF-VNL tasks.}

\section{Purpose and Scope}

We define here a change control process, which applies to any future proposed modifications to the approved technical, cost, and schedule baselines as described in the U.S. Department of Energy (DOE) Office of Fusion Energy Sciences (OFES) HIF Field Work Proposal.

\section{Definitions:}

A baseline is a quantitative description of the Program's technical requirements, costs, and schedule. This baseline serves as the criteria for measuring performance over time. The Baseline Change Control Board is a multidisciplinary group of program representatives designated by VNL management to determine the merit of incorporating proposed changes to program baseline by ensuring the proper definition, coordination, evaluation, and disposition of all baseline change proposals within their area of responsibilities. A Baseline Change Proposal is a document prepared by the initiator of the change. It provides a complete description of the proposed change and its anticipated impacts (positive and negative) on Program baselines. The VNL Baseline Change Proposal Request Form includes the appropriate change request form and the Record of Decision. A Budget Transfer Request is a document used to request changes in baseline funding allocations. A Directed Change is a change imposed on the Project, with direction to implement that affects one or more program baselines. Examples of directed changes include changes to approved budgets or funding and changes resulting from DOE policy directives and regulatory or statutory requirements. An Engineering Change Request is a document that requests changes and provides impacts to baseline scope definition. Thresholds are levels of approval change control authority designated for each level Baseline Change Control Board. The levels are described in Table 4 below. A Schedule Modification Request is a document that requests changes and provides impacts to baseline schedule definition.

\section{Baseline Change Control Process}

The Program uses Baseline Change Control Boards to consider proposed changes to scope, cost, or schedule. Program participants may propose a needed change to the VNL Program baseline. Individuals proposing changes are responsible for discussing the proposed change with the responsible Task Manager and obtaining his/her concurrence. Appropriately detailed information shall be gathered to support the initiation of a change proposal to the Task Manager.

\section{Process Overview}

There are three Baseline Change Control Boards - Levels 1,2, and 3. Each task area within the VNL has a level 3 Baseline Change Control Board that considers only those Baseline Change Proposals affecting their task area. The level 2 Baseline Change Control Board considers those Baseline Change Proposals relating to all task areas that exceed the level 3 thresholds established by this plan. The governing principle is that any member of the Program may submit a Baseline Change Proposal for consideration and approval. The proposal is first considered at Level 3 and acted upon or forwarded with recommendations to the next higher Baseline Change 
Control Board. Dispensation of Baseline Change Proposals shall be made within 2 weeks for routine requests and within 1 week for time-critical requests. All Directed Change requests shall be addressed within times defined by the urgency of implementation with appropriate action to follow as determined by the VNL Director.

Changes proposed from the VNL working level will be in the form of an Engineering Change Request, Schedule Modification Request, or Budget Transfer Request. Analysis supporting the Engineering Change Request, Budget Transfer Request, or Schedule Modification Request will identify the Program baselines that are affected, and determine which Baseline Change Control Board is appropriate to consider the change, given the thresholds of each level. Each Baseline Change Proposal will contain the complete description, justification, or benefit of the proposed change, technical information required to describe the change, and associated performance, cost, and schedule impacts. Supplemental pages are to be used as necessary to present a clear understanding of the proposal. The chairperson shall distribute the Baseline Change Proposal package to members of the Baseline Change Control Board. Technical, cost, and schedule. If during consideration, any change control threshold is exceeded, the Baseline Change Proposal package will be forwarded to the next higher board. The Chairman of any lower-level board that forwards a Baseline Change Proposal to a higher board will act as the primary interface to that board for the purpose of presenting requests for approval.

\section{VNL Level 2 and 3 Baseline Change Control Boards Roles and Responsibilities}

The VNL has two Baseline Change Control Boards - Level 3 at Task Manager authority, and Level 2 at VNL Director authority. Both VNL Baseline Change Control Boards are responsible for review and disposition of all Baseline Change Proposals generated by program participants. This entails evaluation of the proposed change and a decision to accept or decline the modification to the baseline plan. The Baseline Change Control Boards are comprised of a Chairperson, Secretary, voting, and non-voting members. The Level 2 Baseline Change Control Board members are responsible for participating in all Level 2 Baseline Change Control Board meetings to evaluate proposed changes and provide advise to the VNL Director (as Chairperson of the Board) who is responsible to make the final decision on the dispensation of each Level 2 Baseline Change Proposal.

The Chairperson of the Baseline Change Control Board is responsible for appointing a Secretary, voting and non-voting members, and convening and presiding over Baseline Change Control Board meetings. The Chairperson has approval authority for their respective Baseline Change Proposal actions. The VNL Budget Analyst shall be an advisory member of all VNLlevel boards to assess financial impact of specific changes and provide guidance to the Baseline Change Control Board.

The Secretary is responsible to maintain a log of all Baseline Change Proposal actions, a contingency log, and forwarding Baseline Change Proposals exceeding their respective authority level for action at the next higher level. 


\section{VNL Baseline Change Control Board Membership}

Level 3 Baseline Change Control Board (Task Manager)

Chair

Vice-Chair

Secretary

Voting Members

Non-voting Members
Task Manager

Deputy Task Manager

as appointed by Chair

Chief Scientist and Engineer

Program Control Representative

Other scientific/technical members as required

Level 2 Baseline Change Control Board (VNL Director)

Chair

Vice-Chair

Secretary

Advisory Members
VNL Director

VNL Deputy Director appointed by Chair

as appointed by Chair

All other VNL Deputy Directors

Chief Scientist and Engineer

Program Control Representative

Other scientific/technical members as required

The Chair may appoint additional advisors from the VNL program, other agencies, or contractors as deemed necessary for the review.

\section{Level 1 (DOE) Baseline Change Control Board Responsibilities}

The Level 1 Baseline Change Control Board is responsible to review and dispose of all Baseline Change Proposals submitted by the VNL Level 2 Baseline Change Control Board. Actions may include requesting more information, disapproving, or approving Baseline Change Proposals changes within the Level 1 authority threshold.

\section{Baseline Change Thresholds}

The authority of the VNL Baseline Change Control Board is aligned with that defined in the VNL Program Execution Plan. The change thresholds are defined in Table 4. 
Table 4. Baseline Change Control Thresholds.

\begin{tabular}{|l|l|l|l|}
\hline & $\begin{array}{c}\text { DOE Office of the } \\
\text { Fusion Energy Science } \\
\text { (Level 1) }\end{array}$ & \multicolumn{1}{|c|}{$\begin{array}{c}\text { VNL Program } \\
\text { Office } \\
\text { (Level 2) }\end{array}$} & $\begin{array}{l}\text { VNL Task Manager } \\
\text { (Level 3) }\end{array}$ \\
\hline \hline $\begin{array}{l}\text { Technical } \\
\text { (Scope) } \\
\text { Baseline } \\
\text { Thresholds }\end{array}$ & $\begin{array}{l}\text { Any deviation from the } \\
\text { program deliverables as } \\
\text { outlined in the Field Work } \\
\text { Proposal. }\end{array}$ & $\begin{array}{l}\text { Any deviation from scientific } \\
\text { deliverables. }\end{array}$ & $\begin{array}{l}\text { Any deviation from system } \\
\text { design requirements that do } \\
\text { not affect scientific } \\
\text { deliverables. }\end{array}$ \\
\hline $\begin{array}{l}\text { Schedule } \\
\text { (Milestone } \\
\text { Baseline } \\
\text { Thresholds }\end{array}$ & $\begin{array}{l}\text { Changes to Level 1 } \\
\text { milestones in excess of one } \\
\text { year. }\end{array}$ & $\begin{array}{l}\text { Changes to Level 2 milestones in } \\
\text { excess of six months. }\end{array}$ & $\begin{array}{l}\text { Changes to Level 3 } \\
\text { milestones in excess of } \\
\text { three months. }\end{array}$ \\
\hline $\begin{array}{l}\text { Cost (Dollar) } \\
\text { Baseline } \\
\text { Thresholds }\end{array}$ & $\begin{array}{l}\text { Changes greater than } \\
\pm \$ 250 \mathrm{~K} \text { that do not affect the } \\
\text { annual budget. } \\
\text { Changes to the Field Work } \\
\text { Proposal funding profile. }\end{array}$ & $\begin{array}{l}\text { Changes between } \pm \$ 50 \mathrm{~K} \text { and } \pm \$ 250 \mathrm{~K} \\
\text { that do not affect the annual budget. } \\
\text { Changes requiring contingency } \\
\text { allocations greater than } \$ 50 \mathrm{~K} . \\
\text { Changes to distribution of funds } \\
\text { between task areas. }\end{array}$ & $\begin{array}{l}\text { Changes less than } \pm \$ 50 \mathrm{~K} \\
\text { that do not affect the } \\
\text { annual Task budget. }\end{array}$ \\
\hline
\end{tabular}

\section{Documents and Records}

Documents and records generated as a result of implementing the change control process will be generated in a manner suitable for reproduction and signed and dated at the time of completion or approval. Official signed documents will be retained by the VNL Program Document Control Center (DCC). A Document Control Center will be established to retain all supporting documentation regarding the VNL Program. A VNL administrator shall be responsible to assure that all documents that are easily converted to a file format that will not allow modification are archived within the VNL web page. This archive shall be password-protected to prevent public access. All VNL members shall have access to review the archive. The administrator shall archive any documents that are too large (i.e. large blueprints etc.) in a secured filing cabinet. All documents shall be cross-referenced according to subject, VNL document number, and author. 


\section{Enabling Accelerator Technology}

This section describes enabling technology that would be needed for future HIF experiments and for the IRE, but which cannot be funded within this flat budget plan. We include this section to show what is needed if and when additional funding became available. The purpose of accelerator technology $R \& D$ is to

- Enable improved capability and reduce the cost of near-term accelerator experiments.

- Explore the limits of engineering science and materials, to innovate new capability beyond the state-of-the-art in quadrupoles, insulators, core and pulsers.

- Increase the performance and decrease the cost of the IRE and future drivers.

High-gradient, agile waveform induction acceleration modules: Near-term FY02-03 (for IBX) The use of inductive acceleration in IBX requires high voltage cores and pulsers with active waveform control for agile longitudinal beam control. At presently flat OFES funding guidance, we have only $50 \mathrm{~K} / \mathrm{yr}$ to support continued planning and tests in this area, to be ready if and when funding increases for this area. With increased funding, enabling development effort in this area would include the design of a high-gradient induction module test stand. This module will have the flexibility to vary the core and insulator configurations to investigate the limits of increasing the acceleration gradient. Among the goals are to exceed an acceleration gradient of $2 \mathrm{MV} / \mathrm{m}$. Also:

- Continue evaluation of available alloys for IBX induction cores, with an emphasis on cost to deliver volt-seconds, and uniformity of losses sufficient to control waveforms for acceleration control. Efficiency and $5 \mathrm{~Hz}$ pulse rates for cores and switching is desirable but not critical to the IBX mission.

- Evaluation of cost/performance of available insulation coatings and Mylar film insulation techniques for high-voltage, short pulse IBX induction cores, for acceleration gradients $>1 \mathrm{MV} / \mathrm{m}$ average, and to provide ear pulses and longitudinal bunch compression.

- Design a high-gradient induction cell in FY02. Goals are to exceed $1 \mathrm{MV} / \mathrm{m}$ by factors of 2 or more, and to subsequently install the test cell on HCX for acceleration and beam control tests. Test an agile waveform module developed from an SBIR award.

- Assure technical performance, minimize costs, and develop multiple vendors in each technology area.

Long-term (for IRE and drivers) Specific goals for advanced enabling induction acceleration technology to make the IRE and future drivers more affordable and capable are:

1. Pulsers for heavy-ion injectors with fast controllable risetimes, good regulation $0.1 \%$, and 2-4 MV, eventually long pulses of 10-20 $\mu$ s for drivers.

2. Continue working with vendors to improve core material science and manufacturing to produce uniform low loss core material $\left(<2 \mathrm{~kJ} / \mathrm{m} 3 @ 10^{7} \mathrm{~T} / \mathrm{s}\right.$ magnetization rates), for costs which extrapolate to $<5 \$ / \mathrm{kg}$ for $>2000$ ton orders. 
3. Evaluate two high gradient capable insulation schemes (HGI stacks and vacuum/radial) for induction architectures capable of achieving $>2 \mathrm{MV} / \mathrm{m}$ average acceleration gradients @ 100 ns pulses (see figure 7).

4. Working with vendors, design and test all-solid-state pulsers based on IGBT switching using two or three stages of magnetic compression and water-line capacitors, driving 10 induction cellblocks.

5. Integrate the above three elements into an integrated acceleration module driving 10 cells and test efficiency under dummy load @ $5 \mathrm{~Hz}$ for $>10^{8}$ pulses.

Over the near-term, advanced technology will be studied with various small-scale tests to evaluate various feasibility issues. Building the advanced integrated induction acceleration module, possibly including a superconducting magnet array, depends on increased program funding. It would most likely be built after major procurements for the IBX, but still several years before an IRE start.

Figure 7 on the next page shows a conceptual design of an induction acceleration module incorporating the following:

- Low-loss, high flux swing ferromagnetic material.

- Solid-state pulsers for high reliability, long life, and waveform control:

- Power modulator driving parallel cores for the general waveform.

- Regulation modulator driving a separate core for the correction waveform (there is a phase-II SBIR here), incorporating both iterative and feed-forward feedback.

- High-gradient induction cell architecture with low cost insulator.

The beam transport option shown in Figure 7 is a single beam ESQ such as in HCX, but the same acceleration module can be applied to magnetic transport in single beams (such as depicted in Figure 2), or for superconducting magnet arrays for IRE. 


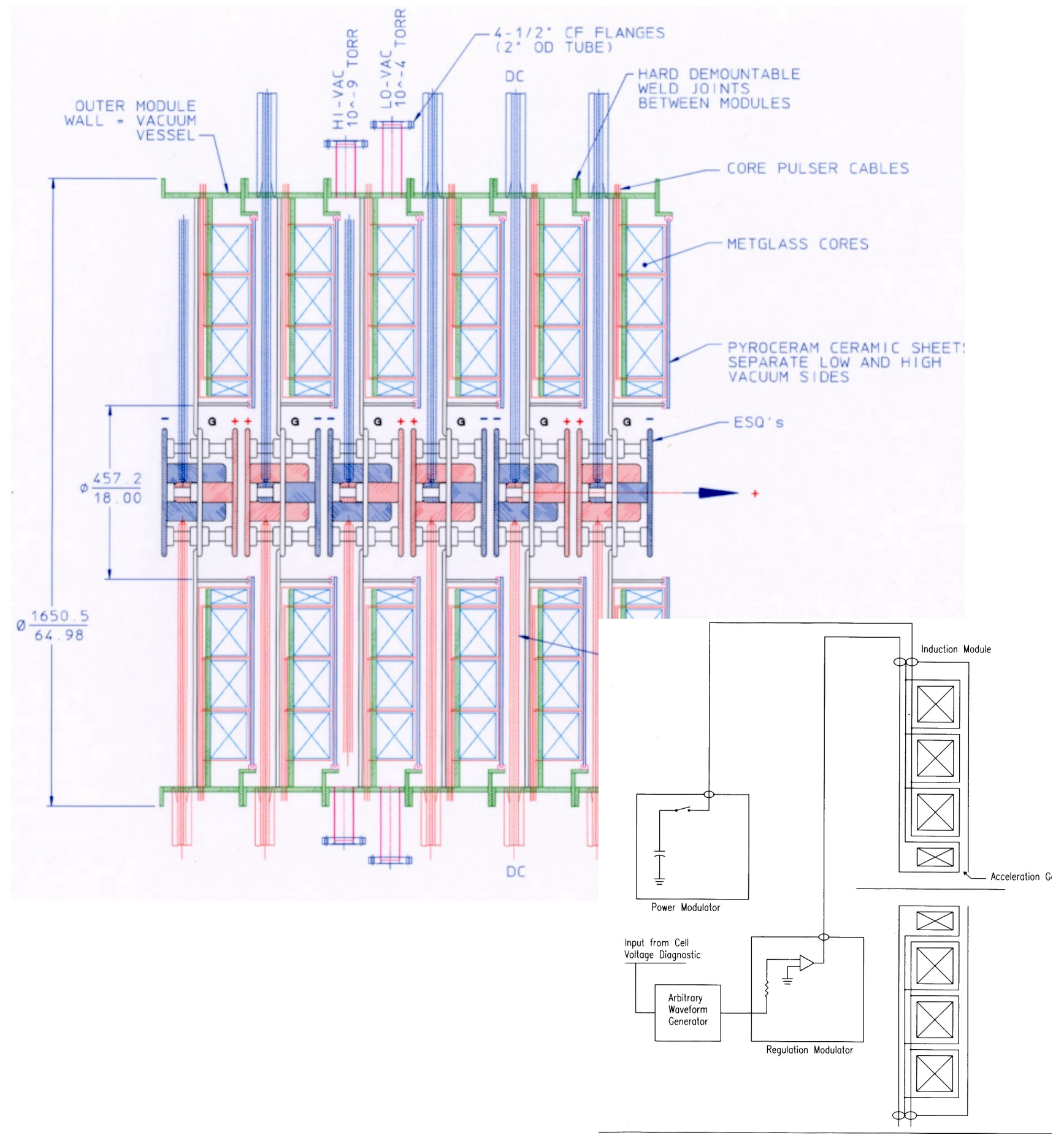

Fig. 7. Conceptual design of a vacuum insulated core for high-gradient, short-pulse induction acceleration, as it might apply to a single beam experiment. Agile waveform control is provided by the circuitry shown in the lower diagram. 